\title{
Multiscale versus micromagnetic calculations of the switching field reduction in FePt/FeRh bilayers with perpendicular exchange spring
}

\author{
F. Garcia-Sanchez ${ }^{\text {a) }}$ and O. Chubykalo-Fesenko ${ }^{\text {b) }}$ \\ Instituto de Ciencia de Materiales de Madrid (CSIC), Cantoblanco 28049, Madrid \\ O. N. Mryasov and R. W. Chantrell \\ Seagate Research, Pittsburgh, Pennsylvania 15222 \\ K. Yu. Guslienko \\ Argonne National Laboratory, Argonne, Illinois 60439
}

(Presented on 9 November 2004; published online 11 May 2005)

Calculations of magnetisation reversal via a perpendicular exchange spring in soft-hard bilayers are presented using the example of an $\mathrm{FePt} / \mathrm{FeRh}$ bilayer which has recently been proposed as an alternative medium for heat-assisted magnetic recording applications. We show that the standard three-dimensional micromagnetic model fails to describe the coercivity reduction due to the fact that at low interface exchange value, the domain wall penetration into the hard layer is not correctly described within the continuous approach. A multiscale model based on atomic level simulations near the interface and a micromagnetic approach far from it is proposed. This leads to improved calculations of the exchange spring at the interface, allowing a detailed study of the magnetisation reversal dynamics. As a result, the coercivity is found to saturate at an interfacial exchange value of $4 \%$ of the bulk value, the practical applications of which are discussed. In addition, the granular structure in an FePt medium with different intergrain exchange parameters is considered. (C) 2005 American Institute of Physics. [DOI: 10.1063/1.1844931]

The FePt/FeRh magnetic bilayer has been recently proposed by Thiele et al. ${ }^{1,2}$ as a potential medium for heatassisted magnetic recording. FeRh belongs to a class of materials with a metamagnetic transition; at room temperature $\mathrm{Fe}_{x} \mathrm{Rh}_{1-x}$ alloy $(x \approx 0.5)$ is antiferromagnetic, undergoing a transition to the ferromagnetic state at temperatures around $300-400 \mathrm{~K}$ depending on the concentration $x$. A remarkable property of $\mathrm{FeRh}$ is that it has a similar lattice parameter to $\mathrm{FePt}$, one of the most promising candidates for future magnetic recording materials. Therefore, the two materials could be grown epitaxially with good interfacial properties. ${ }^{3}$ The advantage of the composite medium is that the antiferromagnetic character of $\mathrm{FeRh}$ at room temperature could provide additional thermal stability, while the coupling between FePt and $\mathrm{FeRh}$ after the metamagnetic transition has occurred could be used to lower the switching field via an exchange spring mechanism. Thiele et al. ${ }^{1,2}$ have measured that a coercivity reduction of $\mathrm{FePt}$ of two to three times could be expected due to this coupling. This reduction of the coercivity is explained by the formation of a new (perpendicular) type of exchange spring: the reversal starts in the soft phase $(\mathrm{FeRh})$, then a Neel-type domain wall is formed which later penetrates into the hard FePt phase and helps reverse its magnetisation. ${ }^{1,4}$ The reduction of the coercivity will depend strongly on the quality of the interface, which can be simulated as a reduction of the interfacial exchange parameter $J_{s}$. Since the possibility of growing a bilayer with an ideal in-

\footnotetext{
${ }^{a)}$ Electronic mail: fgarcias@icmm.csic.es

${ }^{b)}$ Electronic mail: oksana@icmm.csic.es
}

terface is unlikely, it is important for future applications to investigate the switching properties as a function of the reduced exchange interfacial parameter.

The standard approach to describe conventional in-plane "exchange springs" and generalized recently to the case of the perpendicular exchange spring ${ }^{4}$ is a one-dimensional (1D) model. It considers a set of atomistic planes with the properties of FePt or FeRh, each one represented by one magnetic moment. The magnetostatic contribution is taken into account in the demagnetisation field approximation. Our previous analytical and 1D numerical calculations ${ }^{4}$ using this model have shown that the coercivity reduction in the FePt layer due to the exchange coupling to the FeRh layer strongly depends on the interfacial exchange parameter and even for an exchange parameter as low as $10 \%$ of the bulk value, a coercivity reduction of FePt of up to $50 \%$ could be expected.

However, although a 1D approach in the past has been widely used to describe exchange springs and is probably justified by bilayers with a good in-plane film quality, a more general approach is based on full three-dimensional (3D) micromagnetic simulations that correctly take into account the magnetostatic fields. The micromagnetic calculations also provide a means to allow a noncoherent reversal, as well as to consider the granular structure of the film which will be done in the present paper. Our micromagnetic code includes the full magnetostatic contribution using the dynamic alternating direction implicit (DADI) method. ${ }^{7}$ The minimization method was the integration of the Landau-Lifshitz-Gilbert equation with large damping. For FePt we used the following parameters: the anisotropy constant value $K=2$ $\times 10^{7} \mathrm{ergs} / \mathrm{cm}^{3}$ and the saturation magnetisation value $M_{s}$ 


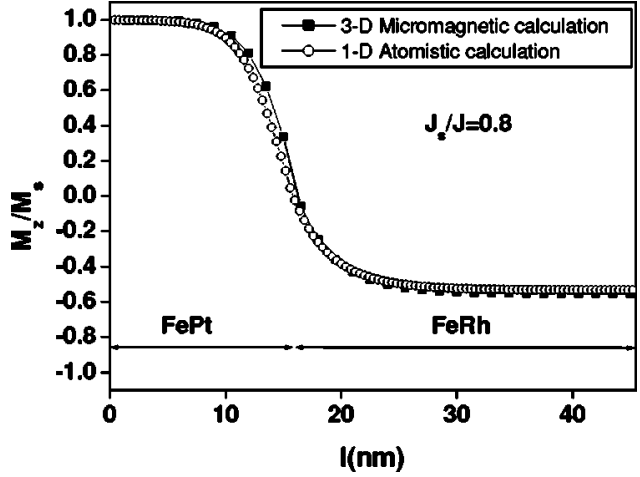

FIG. 1. Comparison of the FePt/FeRh domain-wall width for 3D micromagnetic and 1D atomistic approach for large interface exchange values and for the applied field close to the switching field.

$=1100 \mathrm{emu} / \mathrm{cm}^{3}$, and for FeRh we assumed $K=0$ and $M_{s}$ $=1270 \mathrm{emu} / \mathrm{cm}^{3}$. The exchange stiffness parameter was considered to be the same for FePt and FeRh, $A=10^{-6} \mathrm{ergs} / \mathrm{cm}$. The thickness of the film was assumed to be $15 \mathrm{~nm}$ of FePt and and $30 \mathrm{~nm}$ of FeRh. The discretization length was taken as $1.5 \mathrm{~nm}$, smaller than the exchange-correlation length in FePt, approximately $4.5 \mathrm{~nm}$. The total simulated film had dimensions of $120 \times 120 \times 45 \mathrm{~nm}^{3}$. The boundary conditions were taken as periodic in the film plane. Perpendicular to it, free-space elements were added to simulate zero padding for the magnetostatic potential.

It is clear that the switching properties of FePt will strongly depend on the domain-wall formation at the interface. We have checked that the numerically calculated domain-wall profile in pure FePt has the correct width in comparison to the theoretical prediction. In Fig. 1 we present a comparison between a perpendicular exchange spring [the dependence $M_{z}(z)$ ] calculated within a 1D atomistic approach and 3D micromagnetic simulations when the exchange between the two layers has almost the bulk value. Although the two models give slightly different coercive fields, for the applied fields close to the switching field the domain walls are almost identical. Therefore, our micromagnetic approach correctly describes the domain-wall formation in this (strongly coupled) case.

In the experimental situation the interfacial exchange could be expected to be small in comparison with the bulk exchange value. Our recent results ${ }^{8}$ show that at low interfacial exchange, the domain wall formed in FeRh penetrates into $\mathrm{FePt}$ for several interatomic spacings. This penetration is not present in the micromagnetic approach where the magnetisation undergoes a jump at the interface. Consequently, at reduced exchange values, the micromagnetic approach cannot be expected to describe correctly the switching. For a valid description we use a multiscale model which we recently proposed in Ref. 8. Several micromagnetic discretization units in $\mathrm{FePt}$ and $\mathrm{FeRh}$ are discretized atomistically with the correct FePt and FeRh structures. The exchange interaction inside these "atomistic" cells is taken into account through the Heisenberg model. The exchange interaction between these cells and outer micromagnetic cells are taken into account via the micromagnetic exchange with averaged

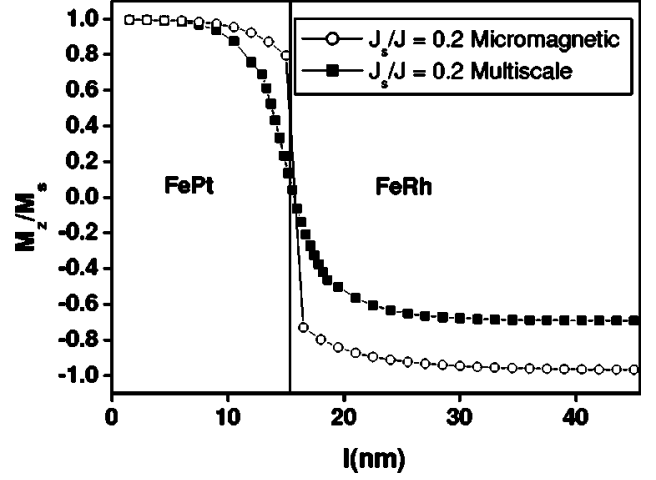

FIG. 2. Comparison between domain-wall structures calculated within micromagnetic and multiscale approach for a small interphase exchange value.

spins from the atomistic cells. The magnetostatic interactions were also evaluated between averaged spins using the DADI approach.

Figure 2 presents a comparison between the micromagnetic domain wall at the $\mathrm{FePt} / \mathrm{FeRh}$ interface and the multiscale approach for a small interfacial exchange parameter value. The difference between the two domain walls is clearly pronounced. The micromagnetic approximation, due to incorrect discretization, does not produce a smooth domain wall. Additionally, it does not predict the penetration of the domain wall into the hard phase which is essentially responsible for the exchange spring effect in the coercivity reduction.

As has been pointed out in Ref. 2, the granular structure is an important feature of an FePt recording medium and must be taken into account in the simulations. To reproduce this realistic feature, we have introduced in our multiscale model "the grains" with a size of $15 \mathrm{~nm}$, grouping together $10 \times 10 \times 10$ micromagnetic discretization units. Since the grain size of $\mathrm{FeRh}$ may be much larger than that of $\mathrm{FePt}$, the FeRh medium was considered to be continuous. The easy axes of the FePt grains were considered to be distributed according to a Gaussian distribution with a small dispersion of $5^{\circ}$. The intergrain exchange parameter $J_{1}$ was assumed to have a reduced value, with respect to the bulk value $J$. Figure 3 represents hysteresis cycles corresponding to small, and intermediate intergrain exchange parameters and continuous FePt medium. The interfacial exchange parameter is also

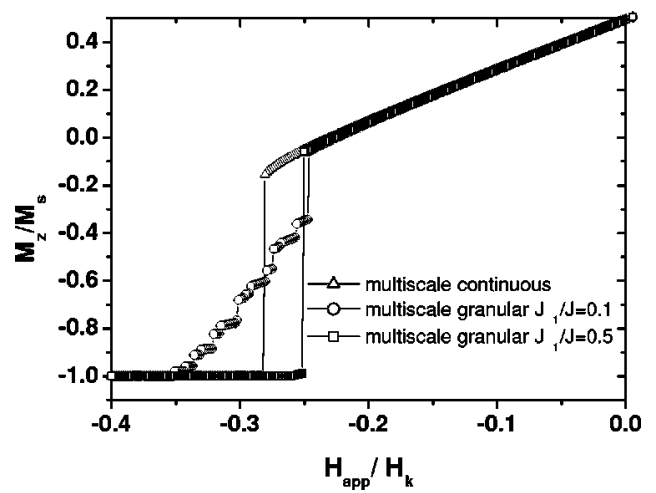

FIG. 3. Simulated hysteresis cycles for granular and continuous media using a multiscale model 


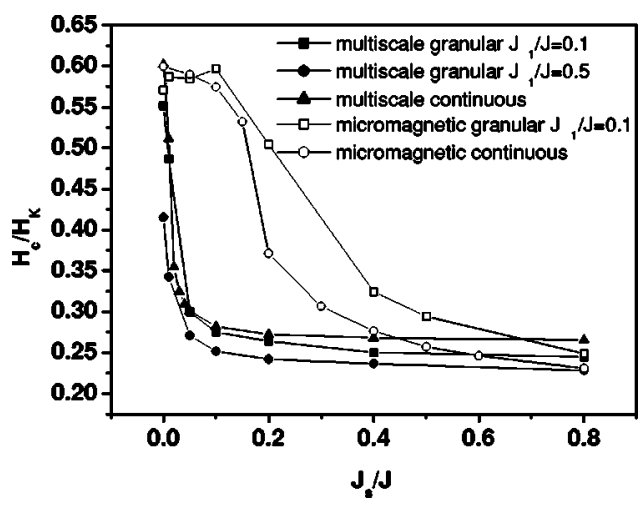

FIG. 4. Coercivity reduction in granular and continuous media within micromagnetic and multiscale approaches.

small in this case. The granular structure introduces additional nucleation centers so that the magnetisation reversal process always starts earlier than that of the continuous medium. In the case of a small intergrain exchange, the reversal process takes place "grain by grain," while for an intermediate intergrain exchange, once started, the reversal process proceeds in one collective reversal.

In Fig. 4 we present the coercivity reduction for a granular and continuum media within the multiscale and micromagnetic approaches. It is clearly observed that the micromagnetic approach shows that in order to achieve a significant coercivity reduction, a large interfacial exchange is necessary. As was pointed out above, this is due to the fact that in the micromagnetic approach the domain wall cannot penetrate into the FePt medium and induce the magnetisation reversal. In contrast, the multiscale approach shows a significant coercivity reduction for interfacial exchange below 5\% of the bulk value. This result is independent of the presence of the granular structure in FePt with or without appreciable integrain exchange. Even if the intergrain exchange is small, the domain wall formed in FeRh penetrates in each grain of FePt, coupling them effectively through the FeRh and producing faster switching.

In conclusion, we have demonstrated that the coercivity reduction in $\mathrm{FePt} / \mathrm{FeRh}$ bilayers is via a complex reversal mechanism involving the penetration of an exchange spring from the soft $(\mathrm{FeRh})$ into the hard $(\mathrm{FeRh})$ magnetic material. For weak interlayer coupling the standard micromagnetic approach cannot reproduce this mechanism. The atomistic model indicates that penetration to the length scale of a few atomic units may be sufficient to induce the magnetisation propagation in a hard material. However, the latter penetration is not allowed in the micromagnetic model due to the lack of discretization. This emphasizes the necessity of atomistic and, in general, multiscale models to describe properly the role of interfaces in the magnetisation reversal process. The result may have a general importance for all cases where the influence of small exchange on the switching properties is considered, such as two-phase permanent magnets. ${ }^{9,10}$ From the point of view of a FePt/FeRh bilayer we have demonstrated that a small amount of interfacial exchange could suffice to decrease the coercivity of FePt by up to $50 \%$. The granular structure of FePt necessary for magnetic recording application does not modify the conclusion, at least provided that the grain size of FeRh is much larger than that of FePt. However, continuous FeRh layers are shown to give rise to an effective exchange coupling within the grains of the hard FePt layer.

The work at ANL was supported by the U.S. Department of Energy, BES Material Sciences under Contract No. W-31109-ENG-38. Two of the authors (O.C.F. and F.G.S.) acknowledge the financial support from Seagate Technology and the Ministery of Science and Education of Spain.

${ }^{1}$ J.-U. Thiele, S. Maat, and E. E. Fullerton, Appl. Phys. Lett. 82, 2859 (2003).

${ }^{2}$ J.-U. Thiele, S. Maat, J. L. Robertson, and E. E. Fullerton, IEEE Trans. Magn. 40, 2537 (2004).

${ }^{3}$ T. Goto, H. Ogata, T. Sato, K. Yamaguchi, T. Shima, and H. Yoshida, J. Magn. Magn. Mater. 272-276, E791 (2004).

${ }^{4}$ K. Yu. Guslienko, O. Chubykalo-Fesenko, O. Mryasov, R. Chantrell, and D. Weller, Phys. Rev. B 70, 104405 (2004).

${ }^{5}$ E. F. Kneller and R. Hawig, IEEE Trans. Magn. 27, 3588 (1991).

${ }^{6}$ A. Hernando, I. Navarro, and J. M. Gonzalez, Europhys. Lett. 20, 175 (1992).

${ }^{7}$ M. R. Gibbons, J. Magn. Magn. Mater. 186, 389 (1998).

${ }^{8}$ F. Garcia-Sanchez, O. Chubykalo-Fesenko, O. Mryasov, K. Yu. Guslienko, and R. W. Chantrell, Appl. Phys. Lett. (submitted).

${ }^{9}$ R. Fischer and H. Kronmuller, J. Appl. Phys. 83, 3271 (1998).

${ }^{10}$ T. Schrefl, J. Fidler, and H. Kronmuller, Phys. Rev. B 49, 6100 (1994). 